\title{
Versatile robotic interface to evaluate, enable and train locomotion and balance after neuromotor disorders
}

\author{
Nadia Dominici ${ }^{1,2,8}$, Urs Keller ${ }^{3,8}$, Heike Vallery ${ }^{3,4}$, Lucia Friedli ${ }^{1,2}$, Rubia van den Brand ${ }^{1,2}$, Michelle L Starkey ${ }^{5,6}$, \\ Pavel Musienko ${ }^{1,2,7}$, Robert Riener ${ }^{3,6}$ \& Grégoire Courtine ${ }^{1,2}$
}

\begin{abstract}
Central nervous system (CNS) disorders distinctly impair locomotor pattern generation and balance, but technical limitations prevent independent assessment and rehabilitation of these subfunctions. Here we introduce a versatile robotic interface to evaluate, enable and train pattern generation and balance independently during natural walking behaviors in rats. In evaluation mode, the robotic interface affords detailed assessments of pattern generation and dynamic equilibrium after spinal cord injury ( $\mathrm{SCl}$ ) and stroke. In enabling mode, the robot acts as a propulsive or postural neuroprosthesis that instantly promotes unexpected locomotor capacities including overground walking after complete $\mathrm{SCl}$, stair climbing following partial $\mathrm{SCl}$ and precise paw placement shortly after stroke. In training mode, robot-enabled rehabilitation, epidural electrical stimulation and monoamine agonists reestablish weightsupported locomotion, coordinated steering and balance in rats with a paralyzing $\mathrm{SCl}$. This new robotic technology and associated concepts have broad implications for both assessing and restoring motor functions after CNS disorders, both in animals and in humans.
\end{abstract}

Neuromotor disorders such as SCI and stroke lead to distinct impairments of motor pattern generation and balance ${ }^{1,2}$. Consequently, dissociating these subfunctions is essential for assessment and neurorehabilitation of gait. Conceptually, neurorehabilitation systems should act as a propulsive or postural neuroprosthesis that assists or perturbs propulsion, balance or the combination of both to varying degrees according to experimental purposes or patient-specific needs.

Existing systems designed to compensate for impaired propulsion and balance rely on passive spring support, counterweight mechanisms or force-controlled systems that generate vertical forces at the trunk level during treadmill-restricted stepping ${ }^{3,4}$. However, these approaches present several drawbacks. These current systems provide support only in the vertical direction, whereas well-balanced locomotion requires finely tuned trunk movements in virtually every direction ${ }^{5}$. Furthermore, the optic flow, which substantially modulates locomotion ${ }^{6}$, is suppressed during treadmill-restricted stepping. Last, rehabilitation is restricted to stepping on a treadmill ${ }^{7}$, a condition that markedly differs from the rich repertoire of natural locomotor tasks.

Robotic systems have been designed to overcome these limitations. The ZeroG ${ }^{8}$ provides vertical support during overground walking using a lifting unit mounted on a rail-guided trolley. However, the rails constrain subjects along a fixed direction, and trunk support is restricted to the vertical direction. The NaviGaitor ${ }^{9}$ allows translations in all directions by means of an overhead linear multiaxis system, but its massive structure leads to high inertia that prevents normal-paced movements.

Here we introduce a multidirectional trunk support system that solves these various issues. The robotic interface continuously and independently assists or perturbs propulsion and balance along four degrees of freedom while rats are progressing overground within a large workspace. Using various models of SCI and stroke, we document advanced capacities to evaluate, enable and train pattern generation and balance during walking under natural conditions encompassing a broad spectrum of locomotor behaviors.

\section{RESULTS}

Design and properties of the robotic interface

We developed a multidirectional support system that exploits three advanced robotic principles (Supplementary Fig. 1). (i) We designed a large serial robotic module consisting of three translational axes defining a Cartesian frame $(x, y, z)$, as well as one rotational axis ( $\varphi$, Fig. 1a). (ii) A parallel Delta linkage prevents tilting and allows measurement of the rat's position. (iii) To decouple the inertia of the massive robotic structure from the end effector, we fabricated a suspension system with a spring assembly that generates forces directed in each of the four degrees of freedom of the serial structure (Fig. 1a). This suspension system capitalizes on the high performance of series elastic actuators for the realization of transparently behaving haptic devices ${ }^{10,11}$. Together, this unique robotic arrangement allows real-time control of horizontal body translations (propulsion, lateral balance) and body-weight support (BWS) (balance) along four independent degrees of freedom that can be

\footnotetext{
${ }^{1}$ Neurology Department, University of Zurich, Zurich, Switzerland. ${ }^{2}$ Center for Neuroprosthetics and Brain Mind Institute, School of Life Science, Swiss Federal Institute of Technology, Lausanne, Switzerland. ${ }^{3}$ Sensory-Motor Systems Lab, Institute of Robotics and Intelligent Systems, Swiss Federal Institute of Technology, Zurich, Switzerland. ${ }^{4}$ Department of Biomedical Engineering, Khalifa University, Abu Dhabi, United Arab Emirates. ${ }^{5}$ Brain Research Institute, University of Zurich, Zurich, Switzerland. ${ }^{6}$ Spinal Cord Injury Center, Balgrist University Hospital, University of Zurich, Switzerland. ${ }^{7}$ Pavlov Institute of Physiology, St. Petersburg, Russia.

${ }^{8}$ These authors contributed equally to this work. Correspondence should be addressed to G.C. (gregoire.courtine@epfl.ch).
} 
a

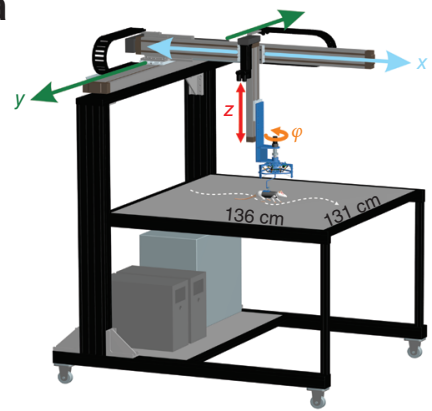

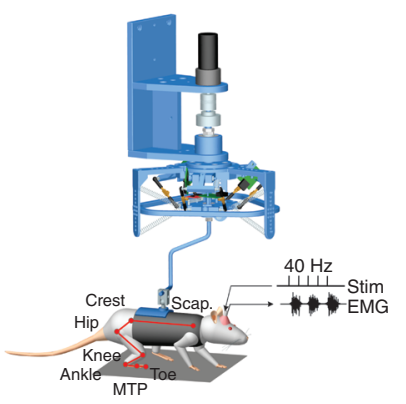

d

b

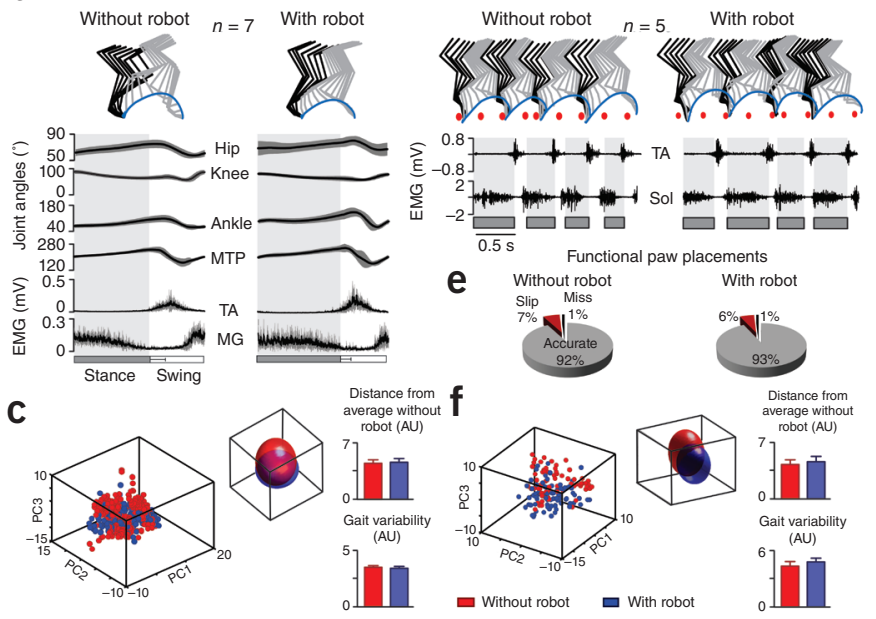

continuously adjusted, that is, from stiff position control to high-fidelity, zero-force control (Supplementary Video 1).

To demonstrate the transparency of the robot, we compared the kinematics and muscle activity underlying locomotion of healthy rats $(n=7)$ walking along a straight runway with and without the robot (Fig. $\mathbf{1 b}$ and Supplementary Fig. 2). Despite detailed analyses (Supplementary Fig. 3), we did not detect significant differences between these conditions $(P>$ 0.3 , Fig. 1c), indicating that the massive robot did not interfere with gait. We confirmed these results during walking on a horizontal ladder $(n=5)$ (Fig. 1d). Even in such challenging conditions, precise paw placement $(P$ $>0.4$, Fig. 1e) and gait features were virtually unaffected by the robotic interface $(P>0.3$, Fig. 1f).

\section{Evaluation of motor pattern generation and balance}

We first aimed to document advanced robot-assisted capabilities to assess motor pattern generation and balance. We embodied these control schemes under the term 'evaluation mode'. Most BWS systems rely on passive spring mechanisms, which provide a support against gravity that is proportional to the subject's vertical position. Although special kinematic configurations can achieve position-independent, constant force support ${ }^{3}$, these passive systems do not compensate for rapid movements. Our robotic system can apply well-controlled, arbitrary vertical force profiles capable of emulating spring-like conditions or a reduced gravitational environment. We exploited this advanced functionality to compare the effect of spring-like versus constant-force BWS conditions on locomotor pattern generation in rats with complete SCI $(n=5$, Fig. 2). To enable stepping, we applied a combination of epidural electrical stimulation and monoamine agonists ${ }^{1}$ (Fig. 2a,b). We tuned the spring constant to an optimal value for facilitating stepping ${ }^{1}$ and maintained the exact same amount of support during constant-force conditions. Compared to spring-like BWS, the constant-force BWS markedly improved the qual-
Figure 1 Design and transparency of the robotic interface in healthy rats. (a) Large and detailed view of the robotic interface. The actuated degrees of freedom are represented with distinct colored arrows. The rat is placed in a skin-like jacket attached to a back plate at the trunk level. EMG, electromyograph; MTP, metatarsophalange. (b) Stick diagram decomposition of hindlimb motion during stance (black) and swing (gray) together with limb endpoint trajectory, hindlimb joint angles and EMG activity of medial gastrocnemius (MG) and tibialis anterior (TA) muscles during locomotion along a straight runway without and with robotic support. (c) Principal component (PC) analysis was applied on all the measured variables $(n=144)$ from all the rats. Gait cycles are represented in the new threedimensional (3D) space created by the three first PCs (explained variance, $39 \%$ ). Least-squares spheres are traced to emphasize the overlap between gaits performed without and with robot. The bar graphs report the average ( $n=7$ rats) 3D distance between conditions (distance for each rat from mean of all gait cycles without robot) as well as 3D dispersion (gait variability). AU, arbitrary units. (d) The same representation is shown during locomotion along irregularly spaced rungs (red dots) with and without robotic support. Sol, soleus. (e) The pie charts show the percentage of steps that were accurately placed versus when the paw missed or slipped off the rung. (f) PC analysis of gait during locomotion along a ladder. Error bars, s.e.m.

ity and consistency $(P<0.01$, Fig. $2 \mathbf{b}, \mathbf{c})$ of gait features (Supplementary Fig. 4) and promoted locomotor patterns that converged toward those of healthy rats $(P<0.01$, Fig. $2 c$ and Supplementary Video 2$)$.

The human ${ }^{12}$ and rat ${ }^{1,13}$ lumbosacral spinal cord can interpret weightbearing information during stepping. We evaluated whether weightbearing input also determines gait quality in rats with complete SCI $(n=4)$. Decreasing the level of constant-force BWS resulted in graded adjustments in hindlimb kinematics, forces and muscle activity $(P<$ 0.01; Supplementary Fig. 5b-e), which confirmed the ability of lumbosacral circuitries to transform weight-bearing information into specific locomotor patterns (Supplementary Video 2). However, we found an inverted U-shaped relationship $\left(R^{2}=0.87\right.$, Supplementary Fig. $\left.5 \mathrm{c}\right)$ between gait quality and the level of BWS. These findings emphasize the importance of optimal constant-force support conditions to enable and train locomotion in subjects with gait disorders.

A unilateral cortical stroke has limited impact on basic locomotion in rats, but behavioral observations have suggested deficits in balance control $^{14}$. To demonstrate impairment of equilibrium after stroke, we exploited the capacity of the robot to superimpose any force at any time and in any actuated degree of freedom onto the zero-force control mode. Specifically, we applied a sudden triangular force profile (2.5 N, Fig. 2d,e) in the mediolateral direction ( $y$ axis, pushing rightward) for $1 \mathrm{~s}$ while rats were progressing freely along a straight runway. Shortly after a left-sided stroke $(6 \mathrm{~d})$, the rats failed to compensate for the perturbation. They showed ample rightward deviations $(P<0.002$, Fig. 2 e,f) and frequently fell off the runway $(56 \% \pm 39 \%$, mean \pm s.d.). After 1 month of recovery, the rats responded to the perturbation with a controlled co-activation of extensor and flexor muscles (Fig. 2g,h), followed by a prolonged activity of contralesional extensor muscles $(360 \% \pm 80 \%, P<0.001$, Fig. 2g,h). This muscle synergy stabilized the trunk and hindlimb (Fig. 2e,f) and produced substantial mediolateral forces $(P<0.001,0.60 \pm$ $0.07 \mathrm{~N}$ at $6 \mathrm{~d}$ versus $1.54 \pm 0.18 \mathrm{~N}$ at $30 \mathrm{~d}$ post-lesion) that restored the locomotor trajectory (Supplementary Video 3). Collectively, these results demonstrate that the evaluation mode of the robotic interface provides heuristic conditions to assess motor pattern generation and balance in neuromotor disorders.

\section{Robot-enabled motor control after neuromotor disorders}

We next sought to use the robotic interface as a propulsive or postural neuroprosthesis that provides adjustable assistance to propel the body forward and to restore postural orientation and stability. We hypoth- 
a

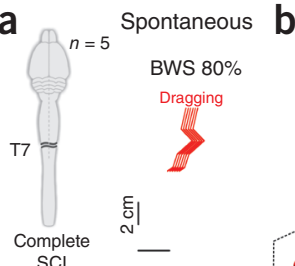

TA 옹리

MG $\div \vec{\circ}: \vec{k}$

Force ₹
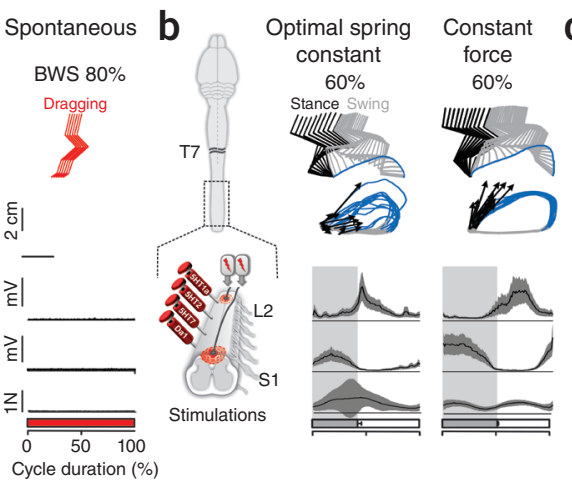

C Distance from intact (AU)

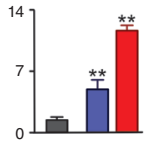

Gait variability $(\mathrm{AU})$

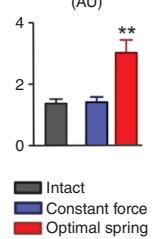

d

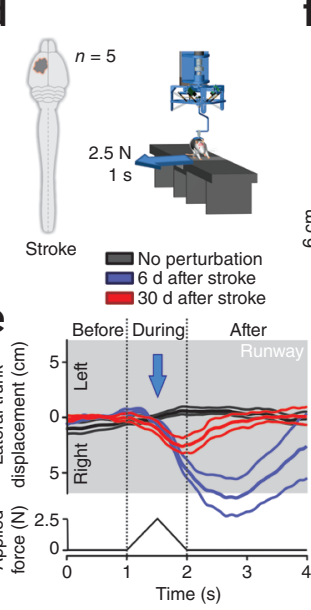

f

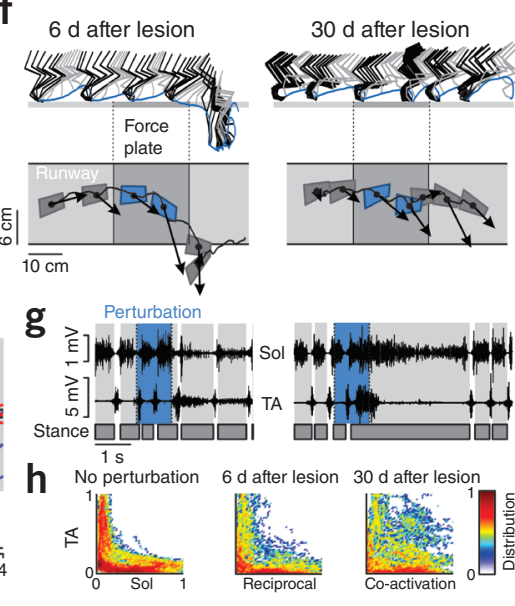

esized that this so-called 'enabling mode' would uncover unexpected locomotor capacities that are dissimulated by impairments in propulsion, balance or both.

Electrical and pharmacological stimulations enable locomotion in rats with complete SCI (Supplementary Fig. 6a,b), but the rats fail to produce the necessary forces to propel their bodies forward overground. Instead, they show tonic activity in extensor muscles, behaviorally apparent as standing (Supplementary Fig. 6c). To com-
Figure 2 The robotic interface affords detailed assessment of pattern generation and balance. (a,b) Rats received a complete SCI. Hindlimb locomotion is shown without (paralysis) (a) and with (b) electrical and pharmacological stimulations. Stick diagram decomposition of hindlimb motion with spring-like versus constant-force BWS is shown together with successive limb endpoint trajectories ( $n=10$ steps), activity of TA and MG muscles and vertical ground reaction forces. The vectors indicate the direction and intensity of foot velocity at swing onset. (c) The bar graphs report the average distance from intact rats as well as gait variability computed through PC analysis. (d) Scheme showing lateral (rightward) postural perturbation ( $1 \mathrm{~s}$ ) applied during runway locomotion in rats with a left-sided cortical stroke. (e) Averaged ( $n=5$ rats) lateral trunk displacements $1 \mathrm{~s}$ before, during and $2 \mathrm{~s}$ after perturbation. (f) Stick diagram decomposition of hindlimb motion and trunk positioning. Arrows indicate the intensity (velocity) and direction of trunk motion. Blue, during perturbation. (g) EMG activity of Sol and TA muscles during a representative trial at $6 \mathrm{~d}$ and $30 \mathrm{~d}$ post-lesion. (h) Plots illustrating coordination (density distribution) between Sol and TA muscles without perturbation and during perturbation at $6 \mathrm{~d}$ and $30 \mathrm{~d}$ after lesion (all trials and rats, scale is the same for all the plots). Error bars, s.e.m. ${ }^{*} P<0.01$ compared to all other nonmarked conditions.

pensate for the lack of propulsion, we configured the robot to act as a propulsive neuroprosthesis that moved the rats forward ( $x$ axis, $13 \mathrm{~cm} \mathrm{~s}^{-1}$ ) while providing constant-force vertical support (60\% \pm $10 \%$ of BWS). After we initiated the robotic guidance, the rats smoothly transitioned from quiet standing to continuous locomotion (Supplementary Fig. 6d). Rhythmic movements arrested instantly when the propulsive neuroprosthesis stopped translating the rat forward (Supplementary Video 4).

Rats with unilateral cortical stroke show significant impairments in contralesional paw placement when crossing a horizontal ladder ${ }^{14}$ (Fig. 3). These deficits have been attributed to the loss of visuomotor control, which heavily relies on the damaged motor cortex ${ }^{15}$. We tested the hypothesis that impaired equilibrium maintenance (Fig. 2d-h) may also contribute to the alteration of skilled locomotion after a cortical stroke. We configured the robotic interface to act as a postural neuroprosthesis. In this enabling mode, the robot provided a constant-force support in the vertical direction ( $z$ axis, $27 \% \pm 4 \%$ of BWS) and stiff support in the lateral directions ( $y$ and rotational axes). The robotic postural neuroprosthesis instantly improved the rats' ability to position their contra-
Figure 3 The robotic postural neuroprosthesis enables skilled motor control after cortical stroke. (a) Schemes depicting the stroke lesion and testing paradigm. Rats were evaluated during walking along a ladder with irregularlyspaced rungs. (b) The relative positioning of the contralesional hindpaw with respect to two successive rung positions (red dots) was evaluated over all the trials from all the rats without and with constant-force robot support. The number of occurrences per $5 \%$ bin is reported together with the percentage of accurate, slipped and missed placements, which are color coded as in a. (c) Stick diagram decomposition of hindlimb motion during a trial along the ladder with and without robot. Hindlimb oscillations and EMG activity of TA and Sol muscles are shown at the bottom. (d) PC analysis (explained variance, $28 \%$ ). Accurate steps are dissociated from missed steps to emphasize that the robot increased the percentage of accurate steps but had no influences on locomotor strategy per se. The bar graph reports the average ( $n=5$ rats) 3D distance from prelesion trials. ${ }^{*} P<0.01$ compared to all prelesion conditions. Error bars, s.e.m.

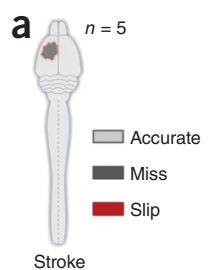

b
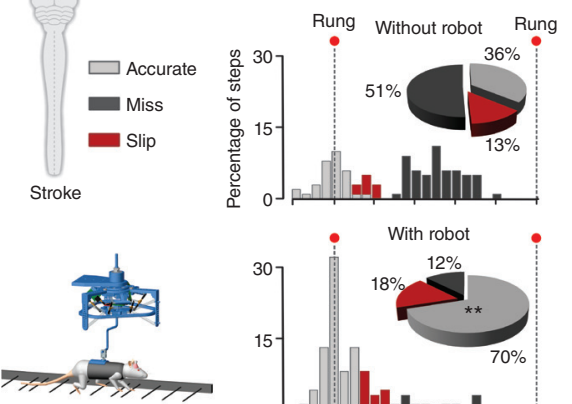

C

Cortical stroke (2 d after lesion)

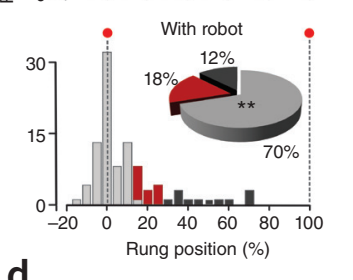

d

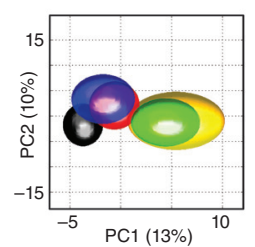

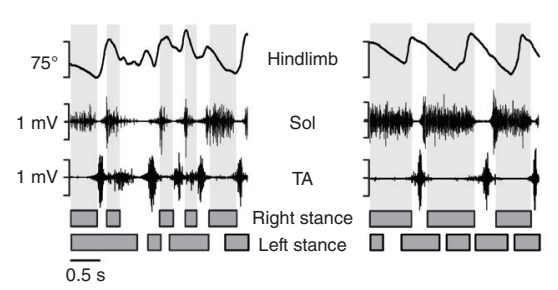

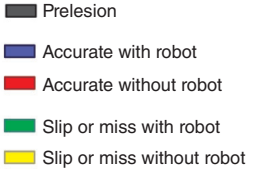

With robot $(25 \%)$
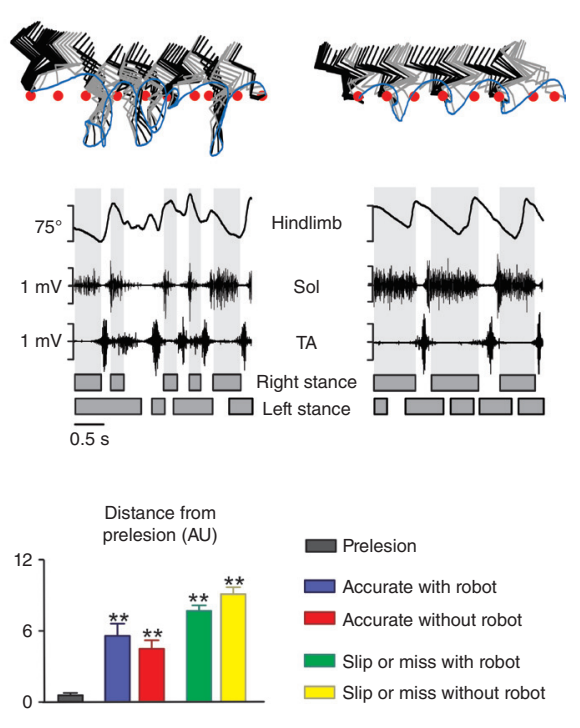

$\square$ Slip or miss without robot 
Figure 4 The robotic postural neuroprosthesis enables coordinated locomotion on a staircase after moderate and severe $\mathrm{SCl}$. (a) Representative stick diagram decomposition of hindlimb motion during climbing on a staircase before lesion. The plots show hindlimb oscillations and EMG activity of MG and TA muscles. (b) Climbing on a staircase without and with constant-force robotic support $10 \mathrm{~d}$ after a $\mathrm{C} 7$ lateral hemisection. (c) PC analysis was applied on all gaits and rats. (d) Percentage of steps accurately positioned onto the staircase. (e) Locomotion on a staircase without and with constant-force robotic support $12 \mathrm{~d}$ after staggered hemisections. Locomotion is shown without (spontaneous) and with electrical and pharmacological stimulations. (f) PC analysis. (g) Percentage of steps accurately positioned onto the staircase. Error bars, s.e.m. ${ }^{* *} P<0.01$ compared to the prelesion condition. a

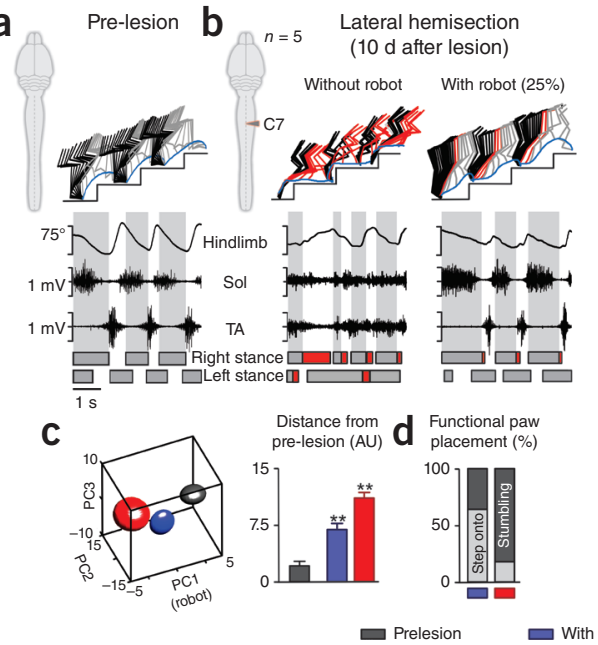

e $n=5 \quad \begin{gathered}\text { Staggered lateral hemisections } \\ \text { (12 dafter lesion) }\end{gathered}$ (12 d after lesion) With robot $(45 \%) \quad$ Without robot $\quad$ With robot $(25 \%)$
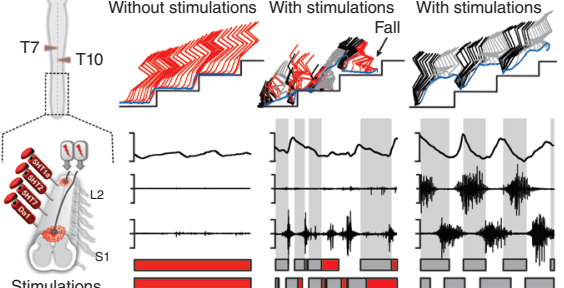
Stimulations
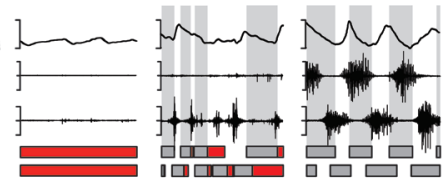

f

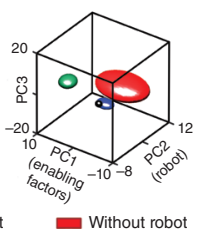

Distance from prelesion (AU) $\mathbf{g} \quad$ Functional paw

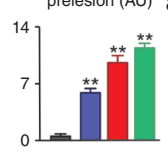
50 - 100 || lesional hindpaws accurately onto the irregularly spaced rungs of the ladder $(P<0.002$, Fig. 3b,c and Supplementary Video 5). Statistical analyses showed that the robot significantly decreased the number of misses and slips $(P<0.01$, Fig. 3d), which correlated with improved postural stability $(P<0.01$, Supplementary Fig. $7 \mathrm{c})$.

We next assessed the capacity of the robotic postural neuroprosthesis to enable motor control in rats with a lateral C7 hemisection $(n=5)$. Ten days after lesion, the rats dragged the ipsilesional hindlimb during locomotion (Supplementary Fig. 8a), especially during climbing on a staircase (Fig. 4). Without robotic support, they stumbled against and rarely stepped onto the staircase (Fig. $\mathbf{4 b}, \mathbf{d}$ ). The robotic postural neuroprosthesis instantly enabled coordinated plantar stepping, both during horizontal walking ( $32 \% \pm 4 \%$ of BWS; Supplementary Fig. 8a,b) and climbing on a staircase $(28 \% \pm 3 \%$ of BWS; Fig. $4 \mathbf{b}, \mathbf{c})$. The robotic support restored trunk orientation and stability $(P<0.001$, Supplementary Fig. 9b,c), which correlated with near-normal hindlimb kinematics (Supplementary Figs. 8 and 9) and accurate positioning of the ipsilesional paw onto the staircase $(P<0.001$, Fig. $4 \mathbf{d}$ and Supplementary Video 6).
We then investigated whether the robotic postural neuroprosthesis could enable motor control shortly after a more severe SCI consisting of two lateral hemisections placed on opposite sides and at different spinal levels (T7 and T10, Fig. 4e). This SCI completely interrupted direct supraspinal input, thus leading to permanent hindlimb paralysis ${ }^{16}$ (Fig. 4e). To enable locomotion as early as $12 \mathrm{~d}$ after SCI, we applied electrical and pharmacological stimulations. Without robotic support, the rats showed rhythmic hindlimb movements, but they failed to perform plantar steps $(91 \% \pm 7 \%$ of dragging, Supplementary Fig. 8e) and often fell laterally during walking (Fig. 4e). With the robotic postural neuroprosthesis, all the tested rats $(n=5)$ showed bilateral weight-bearing plantar steps (Fig. 4e and Supplementary Video 7). Despite the interruption of direct supraspinal pathways, the rats immediately regained the ability to accurately position both hindpaws onto the staircase $(P<0.001$, Fig. 4). The otherwise paralyzed rats showed gait patterns that were nearly indistinguishable from those of healthy rats, both during horizontal locomotion (Supplementary Fig. 8e-h) and stair climbing (Fig. 4e-g). For both tasks, improvement of hindlimb locomotion correlated with robot-enabled recovery of trunk position and stability (Supplementary
Figure 5 Training enabled by the robotic postural neuroprosthesis restores equilibrated steering in rats with a severe $\mathrm{SCl}$. (a) Schemes depicting experimental testing paradigm and conventions to compute body angles. Rats were positioned quadrupedally in the robotic interface, which provided constant-force vertical support while delivering zero force in all the other directions. The rats walked along a $90^{\circ}$-curved runway. Trunk orientation was measured as the angle between the pelvis and the orientation of the upper body velocity vector, termed heading, which also defined the locomotor trajectory. (b) Schemes depicting the SCI, as well as the electrical and pharmacological stimulations for enabling locomotor states. (c) Successive positions of the trunk at swing onset, locomotor trajectory and velocity (arrow's length) of trunk motion as well as direction of upper body motion (arrow) during a representative trial performed before the lesion and at $60 \mathrm{~d}$ after lesion for a nontrained and a trained rat. The bottom plots show the superimposed locomotor trajectories extracted from all the trials of all the rats. The density distribution of locomotor trajectories (all trials and rats) is shown in the top left corner of each plot. (d) Averaged (all rats, \pm s.d.) distance between locomotor trajectories and the optimal trajectory computed from all the prelesion trials ( $n=9$ rats). The shaded area indicates the progression along the curved section of the runway. (e) Averaged distance between each locomotor trajectory and the optimal trajectory. (f) Maximum deviation of the pelvis segment with respect to the heading vector. Error bars, s.e.m. ${ }^{* *} P<0.01$ from all the other nonmarked conditions.

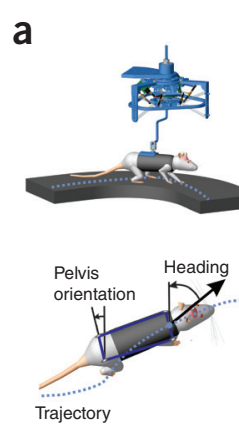

C

Prelesion

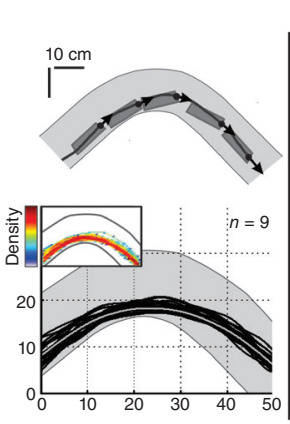

b

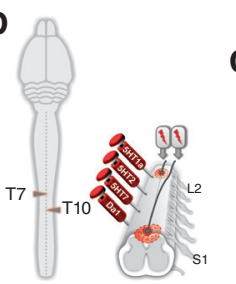

d

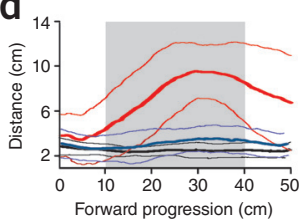

Staggered lateral hemisections (9 weeks after lesion)
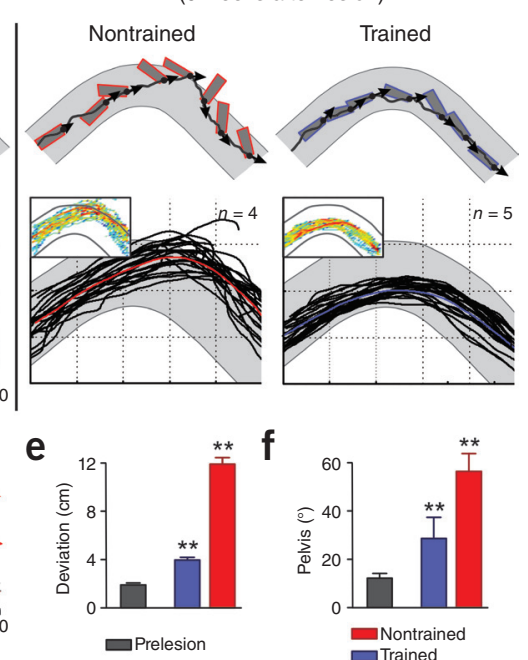
Fig. 10b,c). Together, these findings demonstrate that the enabling mode of the robotic interface instantly restored advanced locomotor capacities across a wide range of natural walking behaviors in moderate to severe neuromotor disorders.

\section{Robot-enabled training after a paralyzing $\mathrm{SCl}$}

Finally, we exploited the enabling mode of the robotic interface to enhance functional capacities with repeated practice, a control scheme that we termed 'training mode. We subjected rats $(n=5)$ with staggered hemisection SCIs to 30-min locomotor training sessions every other day for 8 weeks (see Online Methods). The robotic postural neuroprosthesis provided support against gravity ( $z$ axis), but did not deliver forces in the other directions ( $x, y$ and $\varphi$ axes, Supplementary Fig. 2). Locomotion was enabled by electrical and pharmacological stimulations (Fig. 5). At 9 weeks after lesion, nontrained rats showed weightbearing steps but failed to control body inertia and balance during robot-assisted locomotion along a curved runway $(P<0.001$, Fig. $5 \mathbf{c}-\mathbf{e})$. In contrast, trained rats were capable of steering curves (Fig. $\mathbf{5 c}-\mathbf{f}$ ) while maintaining equilibrated trunk movements $(P<0.001$, Fig. $\mathbf{5 c}, \mathbf{f}$ and Supplementary Video 8). These results reveal that the training mode of the robotic interface markedly improved locomotor capacities in rats with paralyzing SCI.

\section{DISCUSSION}

We have introduced an advanced robotic interface to evaluate, enable and train motor pattern generation and balance across a variety of natural walking behaviors in rats with neuromotor impairments. To hide the inertia of the massive robotic structure, we developed a multidirectional elastic decoupling system that allowed high-fidelity force control. This robotic interface effectively solves the main issues associated with existing rodent and human support systems, such as unidirectional trunk support, high inertia and treadmill-restricted stepping. We validated our methods and concepts in rats with various SCIs and stroke. We expect that similar multidirectional trunk support systems will substantially improve gait rehabilitation in humans with neuromotor disorders.

Evaluation of locomotor function in animals often relies on visual scoring systems ${ }^{17}$ or single-variable analyses ${ }^{14}$ that not only lack objectivity but also fail to capture the multidimensional correlative structures of locomotor control strategies ${ }^{18}$. Here we combined robotically assisted evaluation tools with sophisticated neurobiomechanical and statistical analyses. Together, these paradigms provide the means for assessing the control of, and the interactions between, gait and balance with refinement and objectivity. Future animal and human studies can exploit these analytical tools to evaluate whether and, to a certain extent, how a given therapeutic intervention can enhance specific features of functional recovery in neuromotor disorders.

When acting as a postural or propulsive neuroprosthesis, the robotic interface instantly enabled advanced locomotor capacities in rats with SCI or stroke. We systematically found correlations between robotically restored multidirectional trunk balance and improved hindlimb motor control. These immediate functional improvements emphasize the importance of expanding currently available trunk support systems, which are exclusively unidirectional, to multiple dimensions. Likewise, robotic exoskeletons that provide multidirectional support against gravity enable enhanced upper limb recovery in stroke survivors ${ }^{19}$ and improved locomotion in humans with partial $\mathrm{SCI}^{20}$. These results demonstrate that the concept of robotically enabled motor control has broad implications to enhance functional recovery after CNS disorders.

Our robotic postural neuroprosthesis not only provided multidirectional trunk support but also restored limb and trunk orientation. Consequently, the flow of stretch- and load-related afferent input from hip and ankle joints, which has an essential role in coordinating locomotion $^{21}$, came closer to a normal range. We surmise that the recovery of crucial sensory feedback and its task-specific modulation substantially contributed to reestablishing gait control. For example, the robotic postural neuroprosthesis enabled enhanced hip extension during stair climbing compared to horizontal locomotion. This afferent information appeared sufficient to mediate increased step height and accurate paw placement onto the staircase. Similarly, side-dependent modulation of load- and stretch-sensitive receptors from ankle and trunk muscles during curve walking resulted in the production of asymmetric force patterns that maintained equilibrated steering. These sensorimotor processes were improved with training. Together, these findings confirm and expand current views on the ability of sensory information to act as a source of control for locomotion after the loss of supraspinal influences $^{1,12}$. In this respect, our interface could be equipped with robotic legs attached to the hindlimbs ${ }^{3}$ to ensure appropriate task-specific sensory feedback during rehabilitation ${ }^{22}$.

There is evidence suggesting that gait rehabilitation should be conducted overground ${ }^{23}$ across multiple walking paradigms ${ }^{7}$, with adequate support conditions $s^{23-25}$, enabling systems ${ }^{1,12,19,22,24}$, task-specific sensory cues ${ }^{1,12}$ and active patient cooperation ${ }^{20,22}$, but these concepts remain fragmented. Our versatile propulsive and postural neuroprosthetic interface crystallizes these views into a unified therapeutic tool to evaluate and restore locomotor function after CNS disorders, both in animals and in humans.

\section{METHODS}

Methods and any associated references are available in the online version of the paper.

Note: Supplementary information is available in the online version of the paper.

\section{ACKNOWLEDGMENTS}

We would like to acknowledge the excellent technical help provided by J. Heutschi, N. Wenger, M. Hürlimann, Q. Barraud and S. Duis for data collection and care of the rats, as well as A.S. Tafreshi for the design of the user interface to control the robotic system and A. Brunschweiler, A. Rotta and M. Fritschi for the realization of the suspension system. This work was supported by the National Centers of Competence in Research "Neural Plasticity and Repair" and "Robotics" of the Swiss National Science Foundation, the European Research Council (ERC 261247, "Walk Again”), European Community's Seventh Framework Programme (CP-IP 258654, NeuWALK), the Christopher and Dana Reeve Foundation and the Swiss National Science Foundation (subside 310030_130850).

\section{AUTHOR CONTRIBUTIONS}

U.K., H.V., R.R. and G.C. conceived of and designed the robotic interface. P.M., M.L.S. and G.C. performed the surgeries. N.D. and G.C. conceived of the experiments, analyzed the data and prepared the figures with the help of the other authors. N.D., L.F., R.v.d.B. trained the rats and collected the data. G.C. wrote the manuscript, and all the authors contributed to its editing. G.C. supervised all aspects of the work.

\section{COMPETING FINANCIAL INTERESTS}

The authors declare no competing financial interests.

Published online at http://www.nature.com/doifinder/10.1038/nm.2811. Reprints and permissions information is available online at http://www.nature.com/ reprints/index.html.

1. Courtine, G. et al. Transformation of nonfunctional spinal circuits into functional states after the loss of brain input. Nat. Neurosci. 12, 1333-1342 (2009).

2. Harkema, S.J. et al. Human lumbosacral spinal cord interprets loading during stepping. J. Neurophysiol. 77, 797-811 (1997).

3. Nessler, J.A. et al. A robotic device for studying rodent locomotion after spinal cord injury. IEEE Trans. Neural. Syst. Rehabil. Eng. 13, 497-506 (2005).

4. Frey, M. et al. A novel mechatronic body weight support system. IEEE Trans. Neural. Syst. Rehabil. Eng. 14, 311-321 (2006).

5. Winter, D.A., MacKinnon, C.D., Ruder, G.K. \& Wieman, C. An integrated EMG/biomechanical model of upper body balance and posture during human gait. Prog. Brain Res. 97, 359-367 (1993). 


\section{TECHNICAL REPORTS}

6. Warren, W.H. Jr., Kay, B.A., Zosh, W.D., Duchon, A.P. \& Sahuc, S. Optic flow is used to control human walking. Nat. Neurosci. 4, 213-216 (2001).

7. Musselman, K., Brunton, K., Lam, T. \& Yang, J. Spinal cord injury functional ambulation profile: a new measure of walking ability. Neurorehabil. Neural Repair 25, 285-293 (2011).

8. Hidler, J. et al. ZeroG: overground gait and balance training system. J. Rehabil. Res. Dev. 48, 287-298 (2011)

9. Shetty, D., Fast, A. \& Campana, C.A. Ambulatory suspension and rehabilitation apparatus. US patent $7462138(2008)$

10. Pratt, G.A. et al. Stiffness Isn't Everything. in International Symposium on Experimental Robotics http://citeseerx. ist.psu.edu/viewdoc/summary?doi=10.1.1.26.3136 (1995).

11. Vallery, H. et al. Compliant actuation of rehabilitation robots-benefits and limitations of series elastic actuators. IEEE Robot. Autom. Mag. 15, 60-69 (2008).

12. Harkema, S. et al. Effect of epidural stimulation of the lumbosacral spinal cord on voluntary movement, standing, and assisted stepping after motor complete paraplegia: a case study. Lancet 377, 1938-1947 (2011).

13. Timoszyk, W.K. et al. Hindlimb loading determines stepping quantity and quality following spinal cord transection. Brain Res. 1050, 180-189 (2005).

14. Zörner, B. et al. Profiling locomotor recovery: comprehensive quantification of impairments after CNS damage in rodents. Nat. Methods 7, 701-708 (2010).

15. Drew, T., Andujar, J.E., Lajoie, K. \& Yakovenko, S. Cortical mechanisms involved in visuomotor coordination during precision walking. Brain Res. Rev. 57, 199-211 (2008).

16. Courtine, G. et al. Recovery of supraspinal control of stepping via indirect propriospinal relay connections after spinal cord injury. Nat. Med. 14, 69-74 (2008).
17. Basso, D.M. et al. MASCIS evaluation of open field locomotor scores: effects of experience and teamwork on reliability. Multicenter Animal Spinal Cord Injury Study. J. Neurotrauma 13, 343-359 (1996).

18. Musienko, P. et al. Controlling specific locomotor behaviors through multidimensional monoaminergic modulation of spinal circuitries. J. Neurosci. 31, 9264-9278 (2011)

19. Kwakkel, G., Kollen, B.J. \& Krebs, H.I. Effects of robot-assisted therapy on upper limb recovery after stroke: a systematic review. Neurorehabil. Neural Repair 22, 111-121 (2008).

20. Duschau-Wicke, A., Caprez, A. \& Riener, R. Patient-cooperative control increases active participation of individuals with $\mathrm{SCl}$ during robot-aided gait training. J. Neuroeng. Rehabil. 7, 43 (2010).

21. Peearson, K.G. Generating the walking gait: role of sensory feedback. Prog. Brain Res. 143, 123-129 (2004).

22. Edgerton, V.R. \& Roy, R.R. Robotic training and spinal cord plasticity. Brain Res. Bull. 78, 4-12 (2009).

23. Wessels, M., Lucas, C., Eriks, I. \& de Groot, S. Body weight-supported gait training for restoration of walking in people with an incomplete spinal cord injury: a systematic review. J. Rehabil. Med. 42, 513-519 (2010).

24. Reinkensmeyer, D.J. et al. Tools for understanding and optimizing robotic gait training. J. Rehabil. Res. Dev. 43, 657-670 (2006).

25. Ada, L., Dean, C.M., Vargas, J. \& Ennis, S. Mechanically assisted walking with body weight support results in more independent walking than assisted overground walking in non-ambulatory patients early after stroke: a systematic review. J. Physiother. 56, 153-161 (2010). 


\section{ONLINE METHODS}

Design of the robotic interface. We built a robotic system to provide rats with adjustable trunk support along four independent degrees of freedom. Three actuated linear modules (CKK 20-145, CKK 15-110 and CKK 12-90, Bosch Rexroth AG, distributor: Amsler AG, Feuerthalen, Switzerland) define a large Cartesian workspace capable of translating the rat in $x, y$ and $z$ directions. The first two axes (Fig. 1a, $x$ and $y$ ), which are used for movements in the horizontal plane, cover an area of $1.2 \mathrm{~m}^{2}$. The third axis (Fig. 1a, $z$ ) provides the rat with support against gravity and allows vertical movements over a range of $35 \mathrm{~cm}$. At the extremity of this Cartesian structure, a fourth motor (RE25, Maxon motor AG, Sachseln, Switzerland) actuates rotation $\left(300^{\circ}\right)$ about the vertical axis (Fig. 1a, $\varphi$ ). This serial configuration provides a large workspace in which forces can be applied to the rat while preventing inclinations about the horizontal directions.

We aimed to design a highly versatile robotic system capable of guiding the rats along any desired trajectory that can also behave transparently, that is, allowing the rats to walk freely in the entire workspace without 'feeling' the robot. To obtain this transparency, the interaction forces between the subject and the robot had to be reduced to a minimum. The inertia of the robot (106 $\mathrm{kg}$ in $x$ direction, $32 \mathrm{~kg}$ in $y$ direction, $29 \mathrm{~kg}$ in $z$ direction) is considerably larger than the mass of the rat $(<0.25 \mathrm{~kg})$. Using conventional stiff force sensors and force control, the inertia of the robot could not be hidden from the rat due to theoretical stability limitations to force control ${ }^{26}$. Consequently, a direct coupling between the robot and the subject would yield substantial interaction forces that would interfere with the natural movements of the rat. To hide the inertia of a robotic structure from a substantially lighter interacting subject, Pratt et al. ${ }^{10}$ proposed to couple an actuator to a subject via a compliant element; this configuration is called a series elastic actuator (SEA). Interaction forces and torques can directly be obtained by measuring the deformation of the compliant element that composes the SEA.

However, the concept of SEA has so far only been used for individual actuators, that is, a single degree of freedom. To optimally exploit the SEA concept for our robotic interface, we needed to simultaneously decouple all four actuated modules, requiring that all deformable elements are as close as possible to the rat. To this end, we extended the SEA concept in multiple directions. We conceived a lightweight, low-friction $(<10 \mathrm{~g})$, compliant module consisting of a base platform with three protruding legs forming a cage, a spring-suspended platform within this cage and a Delta linkage that constrains the unactuated degrees of freedom (that is, tilting of the rat) (Supplementary Fig. 1 and Supplementary Video 1). The suspended platform is connected to the cage via six linear springs (angle in the horizontal plane, $120^{\circ}$; stiffness, $112 \mathrm{~N} / \mathrm{m}$ for upper springs, $57 \mathrm{~N} / \mathrm{m}$ for lower springs, Supplementary Fig. 1). An additional spring pair is attached to the rotating shaft in the center of the suspended platform, providing the elastic decoupling about the vertical axis. This configuration decouples the inertia of the serial module from the suspension platform in the four actuated degrees of freedom.

Four contact-free magnetic encoders (12-bit, austriamicrosystems, Austria) are located in the joints of the Delta linkage. These sensors allow measurement of the displacements of the suspended platform, and thereby the deflection of the springs along each degree of freedom, providing an inexpensive way of measuring interaction forces or torques. Four contact-free magnetic encoders (12-bit, austriamicrosystems, Austria) are located in the joints of the Delta structure. The position of the end effector with respect to the serial robot is calculated by combining information from these angular sensors and a forward kinematic model of the Delta linkage (Supplementary Fig. 1). The relative position of the platform encodes the spring lengths and thereby the interaction forces and torques that are derived from the linear spring characteristics.

These forces and torques are used in the force control loop of the robot (Supplementary Fig. 1). The control strategy is implemented in MATLAB/ Simulink and executed in real time on a desktop computer running xPC target (Sampling rate, $1 \mathrm{kHz}$ ). This computer communicates with the motor drives and acquires information coming from the sensors. It also exchanges information with a second computer that runs a user interface for online changes of the control parameters for the robot.

The SEA-based elastic decoupling allows one to set extremely high control gains without affecting stability. The resulting reflected mass of the stiff robot is $787 \mathrm{~g}$ in $x$ direction, $104 \mathrm{~g}$ in $y$ direction, $22 \mathrm{~g}$ in $z$ direction, and $998 \mathrm{~g} \mathrm{~cm}^{2}$ in rotation direction. Owing to the use of the multidimensional SEA, this inertia only dominates the perceived dynamics for low-frequency excitations ${ }^{11}$, for which inertial forces are low. For high-frequency excitations, which are generally associated with reduced amplitudes of motion, the physical properties of the springs dominate the response, also leading to low forces. Consequently, the rat mainly feels the inertia of the suspended platform, which is $109.1 \mathrm{~g}$. The bandwidth of the SEA system is $\sim 2.5 \mathrm{~Hz}$ in $x$ direction, $\sim 2.8 \mathrm{~Hz}$ in $y$ direction, $\sim 13 \mathrm{~Hz}$ in $z$ direction and $\sim 2.2 \mathrm{~Hz}$ in rotation.

Rats and rat care. All procedures and surgeries were approved by the Veterinarian Office Zurich, Switzerland. The experiments were conducted on adult female Lewis rats ( $200 \mathrm{~g}$ body weight, Centre d'Elevage R. Janvier, France). Rats were housed individually on a 12-h light-dark cycle, with access to food and water ad libitum.

Surgical procedures and post-surgical care. All procedures have been described in detail previously $1,16,18$. The surgical interventions were performed under general anesthesia and aseptic conditions. The rats underwent two surgical interventions. First, they were implanted with bipolar intramuscular EMG electrodes (AS632; Cooner Wire, Chatsworth, CA) into selected hindlimb muscles ${ }^{1}$. For some experiments, electrodes also were secured at the midline of the spinal cord at spinal level L2 and S1 by suturing wires (same as EMG wire) over the dura mater above and below the electrode ${ }^{1}$. The rats were allowed to recover for 2 weeks after implantation. After completion of prelesion behavioral recordings, the rats underwent a second surgical intervention during which they received a SCI or a stroke. SCIs included complete transection of the thoracic (T7) spinal $\operatorname{cord}^{1}$, right cervical (C7) lateral hemisection ${ }^{16}$ or two lateral hemisections placed on opposite sides and at different spinal levels (T7 and T10 $)^{16}$. Ischemic lesion to the cortex (stroke) was induced by injecting the vasoconstrictor endothelin-1 $\left(0.3 \mu \mathrm{g}^{-1}\right.$; Sigma-Aldrich) at 14 locations into the left motor cortex (fore- and hindlimb areas). We injected a volume of $500 \mathrm{nl}$ at a depth of $1.2 \mathrm{~mm}$ with a rate of $6 \mathrm{nl} \mathrm{s}^{-1}$. After each injection, the needle was left in place for $3 \mathrm{~min}$ before it was carefully removed ${ }^{14}$. The extent and location of the lesions was verified postmortem. Complete transection SCIs were inspected visually. The extent of the thoracic and cervical hemisections was measured on $40-\mu \mathrm{m}$-thick transverse sections incubated in serum containing anti-GFAP (1:1,000, Z033429, Dako, USA) antibodies. We measured the extent of the lateral lesions over five locations equally spaced over the dorsoventral aspect of the spinal cord. These values were expressed as a percentage of the total mediolateral length and averaged to obtain a unified measure of the lesion extent. Hemisection SCIs ranged from $49.8 \%$ to $54 \%(50.8 \% \pm 0.48 \%)$. In addition, qualitative inspections were carried out to ensure that the lesions conformed to the following specific criteria: (i) minimal sparing of the ipsilesional spinal cord, defined as the absence of white matter sparing, and (ii) minimal damage to the contralesional spinal cord, defined as the near-complete integrity of dorsal and ventral white matter tracts.

Locomotor tasks. A total of seven locomotor tasks were used in the present experiments: bipedal locomotion on a moving treadmill belt $\left(13 \mathrm{~cm} \mathrm{~s}^{-1}\right)$, bipedal walking along a straight runway, quadrupedal walking along a straight runway, lateral perturbation during quadrupedal walking along a straight runway, quadrupedal walking along irregularly spaced round rungs, quadrupedal climbing on a staircase and quadrupedal steering along a $90^{\circ}$-curved runway. The characteristics of the tasks and the dimensions and features of the custom-designed runways can be found in Supplementary Figure 2. The attachment of the rat onto the back plate differed across the tasks and between the various types of injuries. For bipedal locomotion, the rats wore an upper-body jacket that extended from the back of the neck to the iliac crest. The back plate was attached over the entire extent of the jacket via a Velcro strip. For quadrupedal locomotion, the rats wore a whole-body jacket that presented two points of attachment, that is, at the pelvis or at the midthoracic level. The location of the back plate attachment was selected on the basis of the specific gait impairment shown by the rat. Typically, the robot was attached to the pelvis when the rats presented alteration of hindlimb locomotor control, whereas the midthoracic attachment was selected when the rats showed impairment of balance. The dimension of the jackets and location of the attachment pads are reported in Supplementary Figure 2.

Behavioral training of the rats. When the rats first wore the whole-body jacket, they showed changes in their gait pattern. Consequently, the rats were acclima- 
tized to wearing the custom-made jacket for 1-2 weeks while navigating freely along the runways. When no significant difference could be observed between locomotion with and without jacket $(P>0.1)$, we trained the animals daily in one or two sessions until they crossed the runways with a constant speed. Positive reinforcement (food reward) was used to encourage the rats to perform the requested tasks. Rats were trained on the ladder with a regular arrangement of rungs. For testing, rung sequences were irregular and varied to avoid habituation to a particular rung pattern ${ }^{14}$.

Motor control enabling factors. To facilitate locomotion in paralyzed rats, we applied epidural electrical stimulation and a cocktail of monoamine agonists ${ }^{18}$. Rectangular pulses ( $0.2 \mathrm{~ms}$ duration) were delivered at $40 \mathrm{~Hz}$ using two constantcurrent stimulators (AM-Systems, WA, USA) connected to the L2 and S1 electrodes. The intensity of stimulation was adjusted (50-200 $\mu \mathrm{A})$ to obtain optimal facilitation of stepping visually. The rats also received a systemic administration of agonists to 5HT1A/7 (8-OH-DPAT, 0.05-0.1 mg per kg body weight), 5HT2A/C (quipazine, 0.2-0.3 mg per kg body weight) and SKF-81297 (0.15-0.2 mg per kg body weight $)^{18}$.

Testing protocols. Ten step cycles (treadmill) or ten trials (runways) were typically recorded for each rat in a given experimental condition. The conditions with and without robot were randomized across rats. The rats wore the body jacket during walking with and without the robot to maintain the same testing conditions for both types of recordings. When using electrical and pharmacological stimulations to facilitate locomotion, stepping was recorded about $10 \mathrm{~min}$ after drug injection.

Neurorehabilitative training. Rats were subjected to 30 -min training sessions 6 $\mathrm{d}$ per week; starting $12 \mathrm{~d}$ after injury. They were trained for 7 weeks. Locomotion was enabled by electrical and pharmacological stimulations. During each training session, the rats practiced quadrupedal locomotion along the horizontal straight runway, on the staircase and along the $90^{\circ}$-curved runway. We adjusted the respective duration of each task according to the current capacities of the rats. For example, rats only performed a few runs along the curve during each training session until week $4-5$, when they started showing recovery of balance control.

Kinematics and kinetic and EMG recordings. For kinematics, 3D video recordings $(200 \mathrm{~Hz})$ were made using a motion capture system (Vicon, Oxford, UK). Twelve infrared T10 cameras were used to track the motion of reflective markers attached bilaterally at the scapula, iliac crest, greater trochanter (hip), lateral condyle (knee), lateral malleolus (ankle), the distal end of the fifth metatarsal (MTP) and the tip of the toe (Fig. 1a). Nexus (Vicon, Oxford, UK) was used to obtain 3D coordinates of the markers. The body was modeled as an interconnected chain of rigid segments, and joint angles were generated accordingly. The main limb axis termed 'hindlimb' in the figures was defined as the virtual line connecting the greater trochanter to the lateral malleolus.

EMG signals ( $2 \mathrm{kHz}$ ) were amplified, filtered (10-1000 Hz bandpass), stored and analyzed off-line to compute the amplitude, duration and timing of individual bursts ${ }^{1}$. To evaluate temporal coordination between muscles, we generated probability density distributions of normalized EMG amplitudes of agonist and antagonist muscles, as described previously ${ }^{1}$.

Kinetics. Ground reaction torques and ground reaction forces in the vertical, anteroposterior and mediolateral directions were monitored using a force plate ( $2 \mathrm{kHz}$, HE6X6, AMTI, USA) located below the treadmill belt or in the middle of the runway (Supplementary Fig. 2).

Data analyses. A minimum of ten step cycles was extracted for both the left and right hindlimbs for each experimental condition and rat. A total of 148 parameters quantifying gait, kinematics, kinetics and EMG features were computed for each limb and gait cycle according to methods described in detail previously ${ }^{1,16,18}$. These parameters provide a comprehensive quantification of locomotor patterns ranging from general features of gait and performance to fine details of limb motions. The entire list of 148 computed parameters can be found in Supplementary Table 1.

Statistical analyses. The various experimental conditions were associated with substantial modulation of gait patterns, which were evident in the modifications of a large proportion of the computed parameters. To evaluate the more important and reproducible modulation patterns mediated by the different conditions as well as the correlations between the modulated parameters, we implemented a multistep statistical procedure based on PC analysis ${ }^{1}$. The various steps, methods, typical results and interpretation of the analysis are detailed in Supplementary Figure 3. PC analyses were applied on data from all individual gait cycles for all the rats together. Data were analyzed using the correlation method, which adjusts the mean of the data to 0 and the s.d. to 1 . This is a conservative procedure that is appropriate for variables that differ in their variance (for example, kinematic versus EMG data).

All data are reported as mean values \pm s.e.m, unless otherwise specified. Repeated-measures analysis of variance and Student's paired $t$ tests were used to test differences between normally distributed data (Kolmogorov-Smirnov test) from the various experimental conditions. Nonparametric tests (Wilcoxon and Kruskall-Wallis) were used instead when the distribution was not normal.

26. Colgate, E. \& Hogan, N. An analysis of contact instability in terms of passive physical equivalents. Proc. IEEE Int. Conf. Robot. Autom. doi:10.1109/ROBOT.1989.100021 (1989). 